\title{
Estratégias de aprendizagem em curso a distância: validação de uma escala ${ }^{1}$
}

\author{
Thais Zerbini - Universidade de São Paulo \\ Gardênia Abbad - Universidade de Brasília
}

\begin{abstract}
Resumo
Pesquisas em avaliação de treinamentos a distância são incipientes. O artigo pretende contribuir com a área ao construir e validar estatisticamente o instrumento Estratégias de Aprendizagem. O curso-alvo foi oferecido pelo SEBRAE para alunos do Brasil inteiro. A coleta de dados foi realizada ao final do curso por meio de questionário digitalizado que mede a freqüência com que os participantes utilizam as estratégias ao longo do curso. Foram realizadas análises estatísticas de Componentes Principais (PC) e Fatoriais (PAF) e de consistência interna (alfa de Cronbach). Após a validação estatística, o instrumento apresentou sete fatores: Controle da emoção, 5 itens $(\alpha=0,89)$; Busca de ajuda interpessoal, 6 itens $(\alpha=0,89)$; Repetição e organização, 5 itens $(\alpha=0,77)$; Controle da motivação, 4 itens $(\alpha=0,84)$; Elaboração, 3 itens $(\alpha=0,83)$; Busca de ajuda ao material didático, 2 itens $(\alpha=0,75)$; Monitoramento da compreensão, 3 itens $(\alpha=0,82)$. Os resultados indicam que a escala é estatisticamente válida e confiável.

Palavras-chave: Educação a distância; Treinamento; Estratégias de aprendizagem.
\end{abstract}

\section{Learning strategies in distance course: Validation of a scale}

\begin{abstract}
Although few researches on evaluation of distance training. This article intends to contribute on this area when build and statistically validate the instrument Learning Strategies. The evaluated course was offered by SEBRAE to Brazilian students. The data have been collected at the end of the course by questionnaire that measured the frequency with the participants to use the strategies during the course. It have been made statistics analyses of Principal Components (PC) and Factors (PAF) and Internal Consistency (Coefficient Alpha). After the statistical validate, the questionnaire present seven factors: Emotion control, 5 questions $(\alpha=0,89)$; Interpersonal help seeking, 6 questions $(\alpha=0,89)$; Rehearsal and organization, 5 questions $(\alpha=0,77)$; Motivation control, 4 questions $(\alpha=0,84)$; Elaboration, 3 questions $(\alpha=0,83)$; Help of the material, 2 questions $(\alpha=0,75)$; Comprehension monitoring, 3 questions $(\alpha=0,82)$. The results indicated that the scale is valid and reliable statistically.

Keywords: Distance education; Training; Learning strategies.
\end{abstract}

\section{Introdução}

Em razão do aumento da demanda por ações que desenvolvam estratégias de atualização contínua de competências organizacionais, de equipes e individuais, as organizações que compõem o sistema produtivo passaram a desenvolver e oferecer programas de educação corporativa, formação e qualificação profissional mediante a utilização de novas tecnologias de comunicação e informação (NTICs), que possibilitam um alcance maior de pessoas do que cursos presenciais. Diante desse quadro, é fundamental promover a avaliação constante da qualidade e da eficácia das ações educacionais ofertadas para um grande número de pessoas, visando garantir que os objetivos inicialmente propostos sejam alcançados. Entretanto, ainda são raros os estudos sistemáticos na área de psicologia instrucional e organizacional sobre construção e validação de instrumentos avaliativos de tais ações.

\footnotetext{
${ }^{1}$ Apoio: Conselho Nacional de Pesquisa (CNPq).

2 Endereço para correspondência:

Avenida Antônio Machado Sant'Anna, 224 - City Ribeirão Ribeirão Preto SP. 14021-230. E-mail: thais.zerbini@gmail.com
}

O presente artigo pretende contribuir com a área de avaliação de sistemas instrucionais ao propor a construção de itens de medida de estratégias de aprendizagem auto-regulatórias com base no instrumento de Warr e Downing (2000), acrescentando-os à escala de estratégias de aprendizagem, validadas por Zerbini, Carvalho e Abbad (2005), para validação estatística de um novo instrumento. A variável de interesse estratégias de aprendizagem encontra-se inserida no componente características da clientela dos modelos de avaliação de ações de Treinamento, Desenvolvimento e Educação (TD\&E) propostos por Abbad (1999), Baldwin e Ford (1988) e Borges-Andrade (2006).

O componente características da clientela nos modelos de avaliação de Abbad (1999) e Baldwin e Ford (1988) agrupa variáveis motivacionais, cognitivas, demográficas e funcionais dos participantes de ações de TD\&E. No modelo de Borges-Andrade (2006), as características da clientela consistem em fatores físicos e sociais e estados comportamentais associados aos participantes, anteriores à realização da ação educacional, que podem afetar 
seus resultados. Segundo Meneses, Abbad, Zerbini e Lacerda (2006), as variáveis referentes ao componente características da clientela podem ser classificadas de acordo com cinco categorias, a saber: 1. Repertório de entrada: conjunto de conhecimentos, habilidades, atitudes, expectativas e experiências adquiridas pelo participante antes da ação educacional; 2. Sociodemográficas: perfil fisionômico da clientela e perfil profissional e funcional do participante; 3. Psicossociais: variáveis relativas à personalidade, características cognitivas e atitudinais; 4. Motivacionais: motivação para aprender e motivação para transferir; e 5. Cognitivo-comportamentais: estratégias cognitivas, comportamentais e autoregulatórias utilizadas pelo participante para aprender. A variável estratégias de Aprendizagem é classificada, portanto, como características da clientela cognitivo-comportamental.

Em análise da literatura da área de ações educacionais presenciais e a distância (Abbad 1999, Baldwin \& Ford, 1988, Carvalho e Abbad, 2006, Colquitt, LePine \& Noe, 2000, Jegede, 1999, Lacerda \& Abbad, 2003; Meneses \& Abbad, 2004, Zerbini \& Abbad, 2005, entre outros) verificou-se um número reduzido de pesquisas envolvendo aspectos cognitivo-comportamentais. A compreensão de tais características é fundamental para os planejadores instrucionais realizarem escolhas adequadas de estratégias, meios e procedimentos instrucionais ao perfil da clientela. Atualmente, é muito importante, e até mesmo imprescindível, o estudo de tais variáveis no processo de planejamento e programas de educação corporativa a distância, visando ao envolvimento dos funcionários nas ações educacionais propostas e à diminuição dos índices de evasão existentes nesse tipo de curso. Segundo Meneses e colaboradores (2006), com tais informações em mãos, é possível que o profissional de TD\&E elabore estratégias de ensino mais adequadas para que os participantes se beneficiem da ação educacional, evitando, dessa forma, o uso indevido de informações sobre personalidade, motivação, nível de inteligência e aptidões em contextos organizacionais. O estudo de estratégias de aprendizagem pode ser útil para aprimorar o planejamento instrucional de cursos a distância, ao identificar as mais utilizadas pelos indivíduos, bem como ao analisar para quais pessoas são mais adequados determinados procedimentos instrucionais oferecidos.

Segundo Pantoja (2004), a maior parte da produção científica nacional e estrangeira sobre “estratégias de aprendizagem” é proveniente da área da educação, sendo muito reduzido o número de investigações realizadas em contexto de organização e trabalho. Gagné (1968) é um dos principais representantes em estudos sobre o processo de aprendizagem e muito contribuiu para o planejamento da aprendizagem e instrução. Nos estudos clássicos do autor, ele propõe a bierarquia de aprendizagem, a qual representa um conjunto de habilidades intelectuais ordenadas de tal forma que produziriam uma transferência positiva de uma capacidade mais simples para uma mais complexa. Gagné (1972) diferencia seis domínios de aprendizagem e propõe a utilização de tais domínios para distinguir áreas de conteúdos que implicam diferentes tratamentos instrucionais e para elaborar diferentes técnicas de avaliação de resultados de aprendizagem. Os domínios são: 1. Habilidades motoras: capacidades mediadoras e organizadoras de desempenhos motores; 2. Informação verbal: transmissão de fatos, princípios e generalizações; 3 . Habilidades intelectuais: discriminações, conceitos e regras que constituem as habilidades básicas de um currículo elementar e todas as elaborações que ocorrem durante todo o tempo nos mais avançados assuntos; 4. Estratégias cognitivas: habilidades organizadas internamente que orientam o comportamento do indivíduo na aprendizagem, memória e pensamento, e implicam o autogerenciamento da aprendizagem; 5. Atitudes: estado mental interno adquirido que influencia as escolhas de ações pessoais feitas pelo indivíduo; 6 . Enterprise: atividade que depende de combinações de informações verbais, habilidades intelectuais e estratégias cognitivas para ocorrer, estando todas envolvidas com um objetivo comum. As razões para tal classificação incluem a necessidade de avaliação adequada dos desempenhos esperados em uma instrução e a definição de diferentes tipos de eventos para diferentes tipos de domínios, visando à eficácia do evento.

Similar ao conceito de "estratégias cognitivas" proposto por Gagné (1972), Dansereau (1985) define "estratégias de aprendizagem" como um conjunto de processos ou etapas que facilitem a aquisição, a retenção, e a utilização da informação. A definição de Pantoja (2004) consiste em um conjunto de atividades direcionadas ao alcance de metas específicas, quais sejam: aquisição, retenção, recuperação e transferência de novas habilidades e conhecimentos no contexto de trabalho. Verificase similaridade entre os conceitos descritos no que diz respeito às atividades de processamento de 
informações que possibilitam a aquisição, retenção, recuperação e aplicação de conteúdos aprendidos. As definições parecem privilegiar comportamentos e procedimentos utilizados pelos indivíduos para o alcance da aprendizagem bemsucedida.

$$
\text { Warr e Allan (1998) destacam duas }
$$
categorias principais de estratégias de aprendizagem em contexto de organização e trabalho: as estratégias primárias e as autoregulatórias. As primeiras afetam o processo de aprendizagem nas etapas de seleção, armazenamento e recuperação de informações; também são chamadas de estratégias cognitivas por Pintrich, Smith, Garcia e McKeachie (1991). Estratégias auto-regulatórias compreendem a motivação do indivíduo para aprender, a autogestão de esforços e de automonitoramento do progresso durante a aprendizagem, bem como o controle de ansiedade diante de determinadas situações, que estariam dispersando a atenção do aprendiz; também são conhecidas como estratégias de suporte (Dansereau, 1985), afetivas (Weinstein, Zimmerman \& Palmer, 1988) ou de gerenciamento da atenção (Pintrich e colaboradores, 1991).

Apesar de não existir muita discordância entre os pesquisadores quanto à definição de estratégias de aprendizagem, existem conceitos correlatos ao de estratégias que podem gerar dúvidas: hábitos de estudo e estilos de aprendizagem. Warr e Allan (1998) apresentaram importantes diferenciações entre os fenômenos que ajudam a diminuir a confusão conceitual existente. Os autores definem hábitos de estudo como procedimentos utilizados pelo aluno para integrar os melhores aspectos contextuais do ambiente de estudo e maneiras de estudar com o objetivo de melhorar a aquisição e retenção da aprendizagem. São procedimentos focados no contexto e nas atividades de aprendizagem, tais como uso de biblioteca, gerenciamento do tempo de estudo, habilidades de leitura, entre outros. Estilos de aprendizagem são as preferências do estudante referentes aos aspectos contextuais e às maneiras de estudar; compreendem a preferência dos indivíduos por determinados comportamentos de estudo e por aspectos do contexto em que este ocorre. São preferências focadas no contexto e nas atividades de aprendizagem, tais como nível de ruído e temperatura do ambiente ao estudar, horário de preferência de estudo, forma de estudo (individual, em grupo). Segundo Warr e Allan (1998), pesquisas sobre estilos de aprendizagem fornecem evidências que há relação estreita com aspectos da personalidade individual envolvendo atitudes e motivos correlacionados.

Estratégias de aprendizagem são procedimentos (habilidades cognitivas e comportamentais) utilizados pelos indivíduos durante as atividades de aprendizagem de modo a garantir o sucesso de todas suas etapas. São procedimentos focados apenas em atividades de aprendizagem. As estratégias podem ser modificadas por treinamento com o intuito de aumentar a efetividade da aprendizagem em uma atividade ou ambiente específico. Isto significa dizer que não existem estratégias melhores ou piores, mas sim estratégias mais ou menos adequadas ao tipo de atividade a ser aprendida. Segundo Warr e Allan (1998), atividades de diferentes naturezas e graus de complexidades exigem diferentes estratégias de aprendizagem para ocorrerem, portanto, indivíduos em ambiente de trabalho provavelmente utilizam estratégias diferentes de estudantes em ambientes acadêmicos para alcançar a aprendizagem bem-sucedida. Em função dessa preocupação, tais autores desenvolveram um sistema de classificação de estratégias formado por três grandes categorias, as quais compreendem diferentes níveis de análise, a saber: 1) Estratégias cognitivas (primárias): (a) Repetiçãor - repetição mental da informação na forma em que foi apresentada, (b) Organização identificação de idéias centrais do material e criação de esquemas mentais que agrupam e relacionam elementos que foram aprendidos e (c) Elaboração - reflexão sobre implicações e conexões possíveis entre o material aprendido e o conhecimento já existente; 2) Estratégias comportamentais (primárias): (a) Busca de ajuda interpessoal - obtenção de auxílio de outras pessoas, como pares e professores, para tirar dúvidas sobre o material (representa um comportamento proativo do indivíduo de solicitar ajuda), (b) Busca de ajuda no material escrito obtenção de informações em documentos escritos, manuais de instruções, programas de computador e outras fontes que não envolvam contato social, e (c) Aplicação prática - aprimoramento do conhecimento por meio de aplicação prática do que foi aprendido; 3) Estratégias auto-regulatórias: (a) Controle da emoção - controle da ansiedade e prevenção de dispersões de concentração, causadas por sentimentos de ansiedade, (b) Controle da motivação - controle da motivação e da atenção, apesar de existência de um interesse limitado na tarefa a ser aprendida e (c) Monitoramento da compreensão - avaliação do 
processo de aquisição de aprendizagem e modificação do comportamento do indivíduo, quando necessário.

No presente artigo, adotam-se as definições propostas por Warr e Allan (1998) em função dos autores trabalharem com ambientes de trabalho. Portanto, estratégias de aprendizagem, na presente pesquisa, refere-se ao conjunto de capacidades cognitivas complexas aprendidas pelo indivíduo ao longo da vida, o qual envolve capacidades cognitivas, habilidades comportamentais e de autocontrole emocional utilizadas pelo aprendiz para controlar os próprios processos psicológicos de aprendizagem, como atenção, aquisição, memorização e transferência.

Segundo Santos, Boruchovitch, Primi, Zenorini e Bueno (2004), entre os principais instrumentos disponíveis na literatura internacional para mensurar estratégias de aprendizagem em contexto escolar, pode-se destacar o Motivated Strategies for Learning Questionnaire (MSLQ) de Pintrich e colaboradores (1991); e o Learning and Study Strategies Inventory (LASSI), desenvolvido por Weinstein e colaboradores (1988). Santos e colaboradores (2004) e Warr e Downing (2000) construíram e validaram escalas para avaliação de estratégias de aprendizagem baseadas no referencial teórico dos estudos estrangeiros realizados em contexto escolar. O primeiro grupo de autores construiu uma escala voltada para o contexto escolar, enquanto Warr e Downing (2000) desenvolveram uma escala voltada para cursos técnicos, podendo também ser aplicada em contexto escolar.

A escala de Warr e Downing (2000) aborda as nove dimensões propostas por Warr e Allan (1998). O instrumento, em sua versão final, apresentou 45 itens, distribuídos em 8 fatores. O instrumento foi aplicado em duas amostras diferentes para validação, sendo uma composta por participantes de um treinamento em mecânica de veículos $(\mathrm{N}=285)$ e a outra por estudantes universitários $(\mathrm{N}=190)$. Zerbini e colaboradores (2005) desenvolveram um estudo com o objetivo de construir e validar instrumentos de avaliação de treinamentos a distância, entre eles uma escala de estratégia de aprendizagem. As autoras se basearam no instrumento proposto por Warr e Downing (2000), já que esses autores desenvolveram a escala pensando em ambientes de trabalho (cursos técnicos). O questionário de Zerbini e colaboradores (2005), que não continha itens de estratégias auto-regulatórias, apresentou 20 itens em sua versão final, respondidos pelos participantes através de uma escala de 11 pontos do tipo Likert (0-Nunca a 10-Sempre). As respostas dos 1.860 participantes ao questionário foram submetidas à análise dos componentes principais e de fatoração dos eixos principais. O instrumento apresentou uma estrutura com três fatores: Busca de ajuda interpessoal (8 itens, $\alpha=0,85$, cargas fatoriais entre 0,44 e 0,76$)$; Elaboração e aplicação prática do conteúdo (5 itens, $\alpha=0,75$, cargas fatoriais entre 0,38 e 0,83 ); Repetição, organização e ajuda do material (7 itens, $\alpha=0,78$, cargas fatoriais entre $-0,33$ e $-0,69)$.

O fator Busca de ajuda interpessoal (8 itens, $\alpha=0,85)$ encontrado por Zerbini e colaboradores (2005) é similar ao fator de Busca de ajuda interpessoal proposto por Warr e Downing (2000), corroborando, em parte, o modelo teórico e a definição propostos por Warr e Allan (1998). O fator obtido pelas autoras apresentou um índice de confiabilidade semelhante ao de Warr e Downing (2000), que obtiveram um índice de 0,84 e 5 itens. A estrutura empírica encontrada pelas autoras quanto aos fatores Elaboração e aplicação prática do conteúdo e Repetição, organização e ajuda do material não foram similares aos fatores encontrados por Warr e Downing (2000). Os fatores de Zerbini e colaboradores (2005) incluíram itens referentes a estratégias cognitivas e comportamentais em conjunto, e, na estrutura obtida por Warr e Downing (2000), os itens compõem fatores distintos.

$\mathrm{O}$ fato de as estratégias cognitivas e comportamentais se agruparem em um mesmo fator no estudo de Zerbini e colaboradores (2005), pode ter ocorrido em virtude das diferenças no perfil das amostras utilizadas nos estudos, no tipo de cursos e no contexto de aplicação. Warr e Downing (2000) estudaram participantes de cursos presenciais em mecânica de veículos e universitários de cursos técnicos, os quais exigiam muita aplicação prática do conteúdo. Por outro lado, o curso avaliado por Zerbini e colaboradores (2005) foi oferecido a distância pela internet, não exigia aplicação prática do conteúdo, tampouco exigia que o aluno buscasse ajuda no material impresso (a opção de impressão do material era optativa e não-incentivada). Grande parte das diferenças encontradas na escala de Zerbini e colaboradores (2005) e na de Warr e Downing (2000) pode ter sido ocasionada pela utilização do modelo incompleto de estratégias de aprendizagem. A dimensão relacionada a estratégias auto-regulatórias, presente nas propostas de Warr e Allan (1998) e Warr e 
Downing (2000) não foi usada pelas autoras. Borges-Ferreira (2004) adaptou e revalidou a escala de Zerbini e colaboradores (2005) e encontrou uma estrutura semelhante à das autoras: Busca de ajuda interpessoal (6 itens; $\alpha=0,87$ ), Elaboração e aplicação prática do conteúdo (5 itens; $\alpha=0,76)$ e Repetição, organização e ajuda do material (8 itens; $\alpha=0,80)$. Vistos alguns instrumentos de estratégias de aprendizagem existentes na literatura, apresenta-se o método utilizado na pesquisa.

\section{Método}

\section{Participantes}

A pesquisa compreendeu a avaliação do curso Iniciando um Pequeno Grande Negócio (IPGN), ofertado pelo SEBRAE Nacional. O IPGN é gratuito, realizado totalmente a distância via internet, com tutoria ativa e com duração prevista de 40 horas e visa ensinar, passo a passo, a elaboração de um plano de negócios. A população do estudo consistiu no número de inscritos no IPGN no período de 3 de fevereiro a 12 de maio de 2006, totalizando 4.719 alunos. O SEBRAE forneceu os dados sociodemográficos e informações sobre o número de acessos feitos pelos alunos ao ambiente eletrônico do curso. Foram enviados questionários de Estratégias de Aprendizagem a todos os 4.719 alunos e obteve-se um retorno de 993 (21,48\%), o que viabilizou a pesquisa de validação da escala. Verificou-se que a amostra é muito semelhante à população de inscritos no curso. $\mathrm{Na}$ amostra e na população houve um equilíbrio na proporção entre pessoas do sexo masculino e feminino. A maioria reside majoritariamente na região Sudeste, tem nível superior incompleto e possui média de idade de 31 a 33 anos (desvio padrão de aproximadamente 10 anos). Quanto à conclusão do curso, na população, pouco mais da metade o concluiu (66\%), enquanto, nas amostras, o índice de conclusão foi mais alto $(90,7 \%)$. Tanto na população quanto na amostra, foi pouco freqüente $o$ uso das ferramentas da web.

\section{Instrumentos e medidas}

$\mathrm{Na}$ presente pesquisa, optou-se por construir itens de estratégias de aprendizagem autoregulatórias e associá-los aos vinte itens contemplados nos três fatores obtidos por Zerbini e colaboradores (2005), descritos anteriormente. Um dos principais motivos para a construção desses itens consiste na probabilidade de que em cursos a distância, via internet, os participantes tenham que demonstrar um maior controle emocional, motivacional e um maior monitoramento da compreensão para persistir no curso do que em cursos presenciais. O primeiro passo para a construção dos itens foi traduzir os quinze itens de estratégias auto-regulatórias propostos por Warr e Downing (2000) distribuídos em três fatores, a saber: Controle da emoção (5 itens, $\alpha=0,85$ e 0,75$)$, Controle da motivação (5 itens, $\quad \alpha=0,89$ e 0,88$)$, Monitoramento da compreensão (5 itens, $\alpha=0,85$ e 0,84$)$. O instrumento foi aplicado em duas amostras diferentes para validação, sendo uma composta por participantes de um treinamento em mecânica de veículos $(\mathrm{N}=285)$ e a outra por estudantes universitários $(\mathrm{N}=190)$. Em seguida, os itens foram submetidos à análise semântica realizada por alunos de graduação do curso de psicologia da Universidade de Brasília. Após a leitura de cada item, os estudantes os avaliaram quanto à precisão, clareza e objetividade, bem como quanto à adequação das instruções da escala.

\section{Procedimentos}

Coleta de dados

$\mathrm{O}$ instrumento foi hospedado em uma página da internet para a coleta eletrônica de dados. As instruções de preenchimento do questionário foram enviadas por e-mail no último dia de curso. O índice de retorno foi de 21,48\%. As respostas dos participantes ao questionário foram automaticamente registradas em um arquivo de dados eletrônico no programa Excel e, em seguida, importadas pelo SPSS for Windows, versão 13.0, para que pudessem ser analisadas. Pode parecer, a princípio, que o índice de retorno foi baixo. Entretanto, Carvalho e Abbad (2006) e Zerbini e Abbad (2005) obtiveram, em um dos momentos de coleta de dados de suas pesquisas, um total de $8,5 \%$ de retorno. Nesse tipo de coleta de dados a distância, realizada em ambiente não corporativo e em curso gratuito, não se pode esperar altos índices de retorno, como ocorrem em cursos presenciais e corporativos. De qualquer maneira, sabe-se que os índices não foram altos ao ponto de qualificá-los de excelentes. Esse fato pode ser explicado por algumas razões, a saber: alta porcentagem de evadidos do curso (34\%), ausência de vínculo do participante com a instituição e gratuidade do curso. 
Análise de Dados

Foram realizadas análises descritivas e exploratórias para investigar a exatidão da entrada dos dados, a presença de casos extremos, a distribuição dos casos omissos, a distribuição de freqüencia e o tamanho das amostras, de acordo com as orientações de Tabachnick e Fidell (2001). Em seguida, iniciou-se a validação estatística do instrumento. A análise realizada para obter a extração inicial dos fatores foi a dos componentes principais (principal components - PC). Posteriormente, foi realizada análise fatorial (AF). A vantagem de realizar a PC antes da PAF, com todos os seus critérios, é definir os números mínimos de fatores a serem inicialmente extraídos.

\section{Resultados}

As respostas dos 993 participantes aos 34 itens do questionário apresentaram 155 casos extremos univariados e 67 casos extremos multivariados, os quais foram retirados do arquivo de dados, totalizando 771 casos. Foram identificados valores omissos entre 0,3 e $0,9 \%$, e a opção foi não estimar valores para substituir os dados omissos. Algumas características foram analisadas: há mais de 10 casos para cada variável do instrumento; identificou-se a presença de relações lineares entre variáveis; e foram identificados três pares de itens altamente correlacionados entre si, com coeficientes de correlação variando de 0,80 a 0,81 . Após a análise de presença de multicolinearidade, foram retirados da escala dois itens do questionário de Estratégias de Aprendizagem (itens 11 e 12). Para realizar a análise da matriz de covariância em termos de fatorabilidade, foram analisados o tamanho das correlações e a adequação da amostra. Apesar de existirem valores na matriz inferiores a 0,30 , foram identificados, em mais de 50\% dos casos, valores de correlação superiores a 0,30 , indicando que a matriz provavelmente é fatorizável. Quanto ao teste de Kaiser-Meyer-Olkin (KMO), obteve-se um valor de 0,89, considerado por Pasquali (2004) um excelente índice de adequação da amostra.

A análise dos componentes principais, com tratamento pairwise para os casos omissos, sugere uma estrutura empírica com 7 componentes que explicam, em conjunto, $64,42 \%$ da variância total das respostas dos participantes aos itens do questionário. Tal análise seguiu o critério dos eigenvalues (valores próprios) maiores ou iguais a um, e o critério de Harman, no qual cada componente deveria explicar, no mínimo, 3\% da variância total. Assim, poderiam ser extraídos sete fatores, no máximo. Pela análise paralela de Horn, entretanto, observaram-se apenas seis fatores para esta escala (ver Zerbini, 2007). Como a análise do scree plot (distribuição visual de valores próprios) indicou maior ênfase para uma estrutura com sete fatores, foram feitas análises de extração final com 7 e 6 fatores para comparação. As análises foram realizadas por meio da AF, com método de rotação oblíqua e tratamento pairwise para casos omissos. Foram incluídos na escala apenas os itens com conteúdos semânticos similares e cargas fatoriais superiores ou iguais a 0,30 . Na PAF com 6 fatores, dois itens obtiveram cargas acima do critério de $0,30 \mathrm{em}$ dois fatores, 3 itens não permaneceram na escala e as cargas fatoriais eram mais baixas do que na solução com 7 fatores. $\mathrm{Na}$ PAF com sete fatores, apesar de 4 itens não permanecerem na escala, a solução não apresentou compartilhamento de variância entre os itens e as cargas fatoriais apresentaram valores mais altos. Além disso, faz mais sentido teórico uma solução com sete fatores. Portanto, optou-se pela estrutura com sete fatores, assim como indicado na análise dos valores próprios e da variância explicada. A Tabela 1 apresenta a estrutura empírica da escala, as cargas fatoriais, as comunalidades $\left(\mathrm{h}^{2}\right)$ dos itens, os índices de consistência interna das escalas, e os valores próprios e percentuais de variância explicada de cada fator.

Para analisar a estabilidade, verificou-se o quanto os itens eram bons representantes do fator pelo tamanho das cargas fatoriais, as quais apresentaram valores acima de 0,40 . Para analisar a interpretabilidade, ao denominar o fator e associálo ao agrupamento de itens, recorreu-se à análise da literatura da área. Também foi realizada análise de consistência interna. Por último, foi produzido o escore fatorial pela média dos escores das variáveis originais que pertencem ao fator. Quatro itens não alcançaram valor mínimo de carga fatorial 0,30 e não permaneceram na escala.

O fator 1, Controle da emoção, apresentou 5 itens (21 a 25) que correspondem à definição de estratégias de aprendizagem auto-regulatórias, propostas por Warr e Allan (1998), no que tange ao controle da emoção. Esse fator apresentou um bom índice de consistência interna $(\alpha=0,89)$, com cargas fatoriais variando entre 0,48 e 0,91 . O fator 2 , Busca de ajuda interpessoal, apresentou 6 itens (3 a 8 ) que correspondem à definição de estratégias de aprendizagem comportamentais, propostas por Warr e Allan (1998). O fator apresentou um bom índice de consistência interna 
$(\alpha=0,89)$, com cargas fatoriais variando entre 0,66 e 0,83 . O Fator 3, Repetição e organização (5 itens), apresenta um índice razoável de consistência interna $(\alpha=0,77)$ e cargas fatoriais variando entre $-0,35$ e $-0,77$. Os itens que compõem o fator 3 correspondem, na proposta de Warr e Allan (1998), às estratégias cognitivas de repetição (item 14) e de organização (itens 13, 15, 18 e 20). O quarto fator, denominado Controle da motivação, apresentou um total de 4 itens (26 a 29), que correspondem à definição de estratégias de aprendizagem auto-regulatórias, propostas por Warr e Allan (1998). O fator apresentou um bom índice de consistência interna $(\alpha=0,84)$, com cargas fatoriais variando entre 0,69 e 0,86 . O fator 5, Elaboração, inclui 3 itens (16,17 e 19) relativos às estratégias cognitivas de elaboração e apresentou um bom índice de consistência interna $(\alpha=0,83)$, com cargas fatoriais variando entre 0,54 e 0,91 . O sexto fator, denominado Busca de ajuda ao material didático, apresentou apenas 2 itens (1 e 2) que correspondem à definição de estratégias de aprendizagem comportamentais propostas por Warr e Allan (1998). Obteve-se um índice de consistência interna razoável $(\alpha=0,75)$ e cargas fatoriais de 0,68 e 0,82. Finalmente, o fator 7, Monitoramento da compreensão (3 itens - 31, 32 e 33 ), corresponde à definição de estratégias de aprendizagem auto-regulatórias, propostas por Warr e Allan (1998). O fator apresentou um bom índice de consistência interna $(\alpha=0,82)$ e itens com cargas fatoriais variando entre $-0,49$ e $-0,75$.

Do total dos 32 itens analisados, 4 não se agruparam em fator algum, a saber: 9 e 10, referentes às estratégias comportamentais de aplicação prática; e 30 e 34 , referentes às estratégias de monitoramento da compreensão.

Tabela 1. Estrutura empírica da Escala Estratégias de Aprendizagem (continua)

\begin{tabular}{|c|c|c|c|c|c|c|c|c|}
\hline \multirow[t]{2}{*}{ Código/ Descrição dos Itens } & \multicolumn{7}{|c|}{ Cargas fatoriais } & \multirow[t]{2}{*}{$\mathrm{h}^{2}$} \\
\hline & 1 & 2 & 3 & 4 & 5 & 6 & 7 & \\
\hline $\begin{array}{l}\text { 1. Busquei outros sites relacionados ao } \\
\text { conteúdo do curso. }\end{array}$ & & & & & & 0,68 & & 0,52 \\
\hline $\begin{array}{l}\text { 2. Busquei outras fontes de pesquisa, fora da internet, } \\
\text { relacionadas ao curso. }\end{array}$ & & & & & & 0,82 & & 0,70 \\
\hline 3. Expressei minhas idéias nos chats. & & 0,66 & & & & & & 0,45 \\
\hline 4. Expressei minhas idéias na lista de discussão. & & 0,83 & & & & & & 0,68 \\
\hline $\begin{array}{l}\text { 5. Troquei e-mails com meus colegas, participando da } \\
\text { comunidade de aprendizagem. }\end{array}$ & & 0,82 & & & & & & 0,68 \\
\hline $\begin{array}{l}\text { 6. Troquei informações com os colegas sobre o conteúdo do } \\
\text { curso. }\end{array}$ & & 0,74 & & & & & & 0,62 \\
\hline $\begin{array}{l}\text { 7. Troquei informações com os tutores sobre o conteúdo do } \\
\text { curso. }\end{array}$ & & 0,79 & & & & & & 0,63 \\
\hline $\begin{array}{l}\text { 8. Busquei auxílio do tutor para esclarecer minhas dúvidas } \\
\text { sobre o conteúdo. }\end{array}$ & & 0,70 & & & & & & 0,51 \\
\hline 9. Realizei as atividades propostas ao final dos capítulos. & & & & & & & & 0,17 \\
\hline $\begin{array}{l}\text { 10. Revisei os conteúdos relativos aos exercícios em que } \\
\text { cometi erros. }\end{array}$ & & & & & & & & 0,17 \\
\hline 13. Fiz anotações sobre o conteúdo do curso. & & & $-0,77$ & & & & & 0,61 \\
\hline 14. Repeti mentalmente o conteúdo do curso. & & & $-0,42$ & & & & & 0,44 \\
\hline 15. Desenhei esquemas para estudar o conteúdo do curso. & & & $-0,56$ & & & & & 0,52 \\
\hline $\begin{array}{l}\text { 16. Associei os conteúdos do curso aos meus conhecimentos } \\
\text { anteriores. }\end{array}$ & & & & & 0,91 & & & 0,78 \\
\hline $\begin{array}{l}\text { 17. Associei os conteúdos do curso às minhas experiências } \\
\text { anteriores. }\end{array}$ & & & & & 0,91 & & & 0,75 \\
\hline 18. Fiz resumos do conteúdo do curso. & & & $-0,64$ & & & & & 0,57 \\
\hline $\begin{array}{l}\text { 19. Identifiquei, no meu dia-a-dia, situações para aplicar o } \\
\text { conteúdo dos cursos. }\end{array}$ & & & & & 0,54 & & & 0,44 \\
\hline 20. Li o conteúdo do curso no material impresso. & & & $-0,35$ & & & & & 0,22 \\
\hline $\begin{array}{l}\text { 21. Mantive-me calmo diante da possibilidade das coisas } \\
\text { ficarem difíceis. }\end{array}$ & 0,63 & & & & & & & 0,52 \\
\hline 22. Repeti a mim mesmo que tudo sairia bem ao final do & 0,48 & & & & & & & 0,46 \\
\hline
\end{tabular}
curso. 


\begin{tabular}{|c|c|c|c|c|c|c|c|c|}
\hline \multirow[t]{2}{*}{ Código/ Descrição dos Itens } & \multicolumn{7}{|c|}{ Cargas fatoriais } & \multirow[t]{2}{*}{$\mathrm{h}^{2}$} \\
\hline & 1 & 2 & 3 & 4 & 5 & 6 & 7 & \\
\hline $\begin{array}{l}\text { 23. Mantive-me calmo diante da possibilidade de ter um } \\
\text { rendimento no curso abaixo do esperado. }\end{array}$ & 0,85 & & & & & & & 0,67 \\
\hline $\begin{array}{l}\text { 24. Mantive-me calmo diante da possibilidade de cometer } \\
\text { erros ao realizar as atividades do curso. }\end{array}$ & 0,89 & & & & & & & 0,77 \\
\hline $\begin{array}{l}\text { 25. Mantive-me calmo diante da possibilidade das coisas } \\
\text { darem errado. }\end{array}$ & 0,91 & & & & & & & 0,78 \\
\hline 26. Forcei-me a prestar atenção quando me senti cansado. & & & & 0,69 & & & & 0,43 \\
\hline $\begin{array}{l}\text { 27. Esforcei-me mais quando percebi que estava perdendo a } \\
\text { concentração. }\end{array}$ & & & & 0,86 & & & & 0,67 \\
\hline $\begin{array}{l}\text { 28. Aumentei meus esforços quando o assunto não me } \\
\text { interessava. }\end{array}$ & & & & 0,73 & & & & 0,58 \\
\hline $\begin{array}{l}\text { 29. Esforcei-me mais quando percebi que estava perdendo o } \\
\text { interesse. }\end{array}$ & & & & 0,76 & & & & 0,60 \\
\hline 30. Questionei-me sobre o quanto eu havia aprendido. & & & & & & & & 0,40 \\
\hline $\begin{array}{l}\text { 31. Elaborei perguntas para testar minha compreensão sobre } \\
\text { os conteúdos do curso. }\end{array}$ & & & & & & & $-0,75$ & 0,66 \\
\hline $\begin{array}{l}\text { 32. Revisei a matéria para verificar o quanto eu dominava o } \\
\text { conteúdo. }\end{array}$ & & & & & & & $-0,49$ & 0,62 \\
\hline $\begin{array}{l}\text { 33. Elaborei perguntas, testes e provas para estimular minha } \\
\text { aprendizagem. }\end{array}$ & & & & & & & $-0,73$ & 0,69 \\
\hline $\begin{array}{l}\text { 34. Esforcei-me mais para reforçar minha compreensão dos } \\
\text { conteúdos ensinados. }\end{array}$ & & & & & & & & 0,47 \\
\hline $\mathrm{N}$ & 767 & 766 & 762 & 766 & 766 & 768 & 767 & \\
\hline Eigenvalue (valor próprio) & 7,48 & 4,16 & 1,98 & 1,45 & 1,19 & 0,95 & 0,56 & \\
\hline$\%$ da variância explicada & 23,4 & 13,0 & 6,19 & 4,59 & 3,71 & 2,98 & 1,76 & \\
\hline $\mathrm{N}^{\mathrm{o}}$ de itens & 5 & 6 & 5 & 4 & 3 & 2 & 3 & \\
\hline $\operatorname{Alfa}(\alpha)$ & 0,89 & 0,89 & 0,77 & 0,84 & 0,83 & 0,75 & 0,82 & \\
\hline
\end{tabular}

\section{Discussão}

Os índices de confiabilidade dos fatores obtidos variaram de 0,75 a 0,89 , o que caracteriza que a escala apresenta consistência interna. Além disso, as cargas fatoriais variaram de $-0,77$ a 0,91 , indicando que, além de consistente, a escala é válida. Uma das contribuições deste estudo foi a construção de itens de estratégias de aprendizagem auto-regulatórias, os quais foram acrescentados à escala proposta por Zerbini e colaboradores (2005). Tal esforço de pesquisa se justifica em virtude da necessidade de minimizar grande parte das diferenças encontradas entre a escala desenvolvida pelas referidas autoras, revalidada por Borges-Ferreira (2004), e a escala original, desenvolvida por Warr e Downing (2000). Foram obtidos sete fatores de Estratégias de Aprendizagem, os quais apresentam maiores similaridades com as dimensões teóricas propostas por Warr e Allan (1998) e com a estrutura empírica encontrada por Warr e Downing (2000) do que as dimensões e estrutura obtidas nos estudos de Zerbini e colaboradores (2005) e Borges-Ferreira (2004).
O fator Controle da emoção (5 itens, $\alpha=0,89$, cargas fatoriais entre 0,48 a 0,91 ) é similar ao fator de mesmo nome obtido por Warr e Downing (2000), que apresenta um índice de confiabilidade de 0,85 para 5 itens, com cargas fatoriais variando entre 0,66 e 0,72 . O fator Busca de ajuda interpessoal ( 6 itens, $\alpha=0,89$, cargas fatoriais entre 0,66 a 0,83 ) é similar ao fator de mesmo nome obtido por Warr e Downing (2000), que apresenta um índice de confiabilidade de 0,84 para 5 itens, com cargas fatoriais variando entre 0,54 e 0,68. O terceiro fator, denominado Repetição e organização (5 itens, $\alpha=0,77$, cargas fatoriais entre $-0,35$ a $-0,77)$ agrupa itens pertencentes, inicialmente, a duas estratégias cognitivas distintas. Segundo Warr e Allan (1998), as estratégias cognitivas de repetição consistem na repetição mental da informação na forma em que foi apresentada ao aluno, e as estratégias de organização se referem à identificação de idéias centrais do material e criação de esquemas mentais que agrupam e relacionam elementos que foram aprendidos. $\mathrm{Na}$ estrutura obtida por Warr e Downing (2000), as duas estratégias que se agruparam em um único fator foram Organização 
e elaboração, denominado pelos autores de Reflexão ativa, com 10 itens, índice de confiabilidade de 0,90 e cargas fatorias variando de 0,47 a 0,75 .

Do mesmo modo que os fatores Controle da emoção e Busca de ajuda interpessoal, o quarto fator, Controle da motivação (4 itens, $\alpha=0,84$, cargas fatoriais variando entre 0,69 e 0,86), apresentou similaridade com o fator de mesmo nome obtido por Warr e Downing (2000), que apresenta um índice de confiabilidade de 0,89 para 5 itens, com cargas fatoriais variando entre 0,64 e 0,78 . Elaboração, quinto fator obtido no presente estudo, com 3 itens, $\alpha=0,83$ e cargas fatoriais variando de 0,54 a 0,91 , não apresentou a mesma estrutura empírica encontrada por Warr e Downing (2000), a qual agrupou em um único fator, as estratégias cognitivas de aprendizagem de organização e elaboração, denominado Reflexão ativa. Na proposta de Warr e Allan (1998), estratégias de elaboração correspondem aos procedimentos adotados pelo aluno no intuito de analisar e refletir sobre implicações e conexões possíveis entre o material aprendido e o conhecimento e experiências já existentes. O fato dos itens de elaboração, na presente pesquisa, terem se agrupado em um único fator e os itens de Repetição e organização em outro fator pode encontrar explicação na heterogeneidade dos participantes do curso IPGN. Este é aberto e gratuito para qualquer cidadão brasileiro que pretende desenvolver habilidades de empreendedorismo. Os participantes desse curso, portanto, são de diferentes regiões do país e, provavelmente, possuem diferentes experiências profissionais e diferentes expectativas quanto ao curso, diferentemente das amostras utilizadas por Warr e Downing (2000). Diante disso, é provável que os participantes utilizem de forma isolada e com mais freqüência os procedimentos de análise sobre possíveis conexões entre o material aprendido e o conhecimento e experiências já existentes (elaboração) do que os procedimentos de repetição mental do conteúdo e de criação de esquemas mentais que agrupam e relacionam elementos que foram aprendidos (repetição e organização).

O sexto fator, Busca de ajuda ao material didático ( 2 itens, $\alpha=0,75$, cargas fatoriais de $0,68 \mathrm{e}$ $0,82)$, assim como os outros citados anteriormente, também apresentou semelhança com o fator obtido por Warr e Downing (2000), que obteve índice de confiabilidade de 0,89 para 5 itens, com cargas fatoriais variando entre 0,73 e
0,80. E, finalmente, Monitoramento da compreensão, sétimo fator obtido na presente pesquisa, com 3 itens, $\alpha=0,82$ e cargas fatoriais entre -0,49 e -0,75, também foi semelhante à estrutura obtida no estudo de Warr e Downing (2000), com 5 itens, $\alpha=0,85$, e cargas fatoriais entre 0,48 e 0,68 .

Do total dos 32 itens analisados, quatro não se agruparam em fator algum, sendo dois deles referentes a estratégias comportamentais de aplicação prática e dois relacionados a estratégias de monitoramento da compreensão. A razão dos itens relacionados à aplicação prática não terem permanecido na escala pode dever-se ao fato do IPGN não exigir do aluno aplicação prática do conteúdo ao longo do curso, uma vez que os exercícios propostos ao final dos capítulos mediam apenas a compreensão dos conteúdos. $\mathrm{Na}$ estrutura obtida por Warr e Downing (2000), o fator aplicação prática apresentou 5 itens, $\alpha=0,78$, e cargas fatoriais entre 0,56 e 0,75. Sugere-se a construção de mais itens referentes à aplicação prática do conteúdo para serem testados em cursos que apresentem exercícios mais complexos, assim como o curso técnico profissionalizante avaliado por Warr e Downing (2000), o qual exigia bastante aplicação prática do conteúdo. Os itens referentes ao monitoramento da compreensão excluídos da escala na versão final ("Questioneime sobre o quanto eu havia aprendido" e "Esforcei-me mais para reforçar minha compreensão dos conteúdos ensinados") devem ser revistos para as próximas aplicações, pois são muito imprecisos em comparação com os demais itens que compõem o fator.

$\mathrm{Na}$ presente pesquisa, não foram obtidos fatores nos quais estratégias cognitivas e comportamentais se agrupassem em um único fator, tal como ocorreu nos estudos de Zerbini e colaboradores (2005) e Borges-Ferreira (2004). Verifica-se, portanto, a confirmação da necessidade de construir e associar itens de estratégias auto-regulatórias à estrutura existente para que a estrutura das referidas autoras se torne mais similar à de Warr e Allan (1998) e Warr e Downing (2000). Em suma, as escalas de Controle da Emoção, Busca de Ajuda Interpessoal, Controle da Motivação, Busca de Ajuda ao Material Didático e Monitoramento da Compreensão são medidas equivalentes conceitualmente e empiricamente às de Warr e Downing (2000). As estratégias de Repetição e Organização e Elaboração são similares às medidas dos referidos autores. Por fim, recomenda-se que a Escala de 
Estratégias de Aprendizagem desenvolvida neste estudo seja aplicada em outras organizações e em diferentes cursos para revalidação e confecção de estrutura empírica mediante análise fatorial confirmatória.

\section{Referências}

Abbad, G. (1999). Um modelo integrado de avaliação do impacto do treinamento no trabalho - IMPACT. Tese de Doutorado. Brasília: Universidade de Brasília - Instituto de Psicologia.

Baldwin, T. T. \& Ford, J. K. (1988). Transfer of training: A review and directions for future research. Personnel Psychology, 41(1), 63-105.

Borges-Andrade, J. E. (2006). Avaliação integrada e somativa em TD\&E. Em J. E. BorgesAndrade, G. Abbad \& L. Mourão (Orgs.). Treinamento, desenvolvimento e educação em organizações e trabalho: fundamentos para a gestão de pessoas (pp. 343-358). Porto Alegre: Artmed.

Borges-Ferreira, M. F. (2004). Avaliação de Reações $e \quad$ Aprendizagem em disciplinas de curso técnico profissionalizante oferecidas a distância. Dissertação de Mestrado. Brasília: Universidade de Brasília - Instituto de Psicologia.

Carvalho, R. S. \& Abbad, G. S. (2006). Avaliação de treinamento a distância: reação, suporte à transferência e impactos no trabalho. Revista de Administração Contemporânea, 10(1), 95-116.

Colquitt, J. A, LePine, J. A. \& Noe, R. N. (2000). Toward an integrative theory of training motivation: A meta-analytic path analysis of 20 years of research. Journal of Applied Psychology. 85(5), 678-707.

Dansereau, D. F. (1985). Learning strategies research. Em J. W. Segal, S. F. Chipman \& R. Glaser (Orgs.). Thinking and Learning Skills (pp. 209-239). Hillsdale, NJ: Lawrence Erlbaum.

Gagné, R. M. (1968). Learning hierarchies. Educational Psychologist, 6, 1-9.

Gagné, R. M. (1972). Domains of learning. Interchange, 3, 1-8.

Jegede, O. (1999). Differences between low and high achieving distance learners in locus of control and metacognition. Distance Education. 20(2), 255-263.
Lacerda, E. R. M. \& Abbad, G. (2003). Impacto do treinamento no trabalho: investigando variáveis motivacionais e organizacionais como suas preditoras. Revista de Administração Contemporânea, 7(4), 77-96.

Meneses, P. P. M. \& Abbad, G. (2003). Preditores individuais e situacionais de auto e heteroavaliação de impacto do treinamento no trabalho. Revista de Adminstração Contemporânea, 7, ed. especial.

Meneses, P. P. M., Abbad, G, Zerbini, T., \& Lacerda, E. (2006). Medidas de Características da clientela em avaliação de TD\&E. Em J. E. Borges-Andrade, G. Abbad \& L. Mourão (Orgs.). Treinamento, desenvolvimento e educação em organizações e trabalho: Fundamentos para a gestão de pessoas (pp. 422-442). Porto Alegre: Artmed.

Pantoja, M. J. (2004). Estratégias de aprendizagem no trabalho e percepcões de suporte à aprendizagem continua - Uma análise multinivel. Tese de Doutorado. Brasília: Universidade de Brasília Instituto de Psicologia.

Pasquali, L. (2004). Análise fatorial para pesquisadores. Petrópolis: Vozes.

Pintrich, P. R., Smith, D. A. F., Garcia, T. \& McKeachie, W. J. (1991). A Manual for use of the motivated strategies for Learning Questionnaire. Ann Arbor, MI: School of Education, University of Michigan.

Santos, A. A. A., Boruchovitch, E., Primi, R., Zenorini, R. P. C. \& Bueno, J. M. H (2004). Escala de avaliação de estratégias de aprendizagem para universitários (EAP-U): aplicação do Modelo de Rasch de créditos parciais. Braga-PT: Psicologia: Teoria, Investigação e Prática, 9(2), 227-242.

Tabachnick, B.G. \& Fidell, L.S. (2001). Using multivariate statistics. New York: HarperCollins College Publishers.

Warr, P. \& Allan, C. (1998). Learning strategies and occupational training. Internacional Review of Industrial and Organizational Psychology, 13, 83121.

Warr, P. \& Downing, J. (2000). Learning strategies, learning anxiety and knowledge acquisition. British Journal Psychology, 91, 311333.

Weinstein, C. E., Zimmerman, S. A. \& Palmer, D. R. (1988). Assessing learning strategies: the 
design and development of the LASSI. Em C. L. Weinstein, E. T. Goetz \& P. A. Alexander (Orgs.). Learning and Study Strategies (pp. 25-39). San Diego: Academic Press.

Zerbini, T. (2007). Avaliação da transferência de treinamento em curso a distância. Tese de Doutorado. Brasília: Universidade de Brasília Instituto de Psicologia.

Zerbini, T. \& Abbad, G. (2005). Impacto de treinamento no trabalho via internet. Revista de Administração de Empresas Eletrônica, 4 (2).
Zerbini, T., Carvalho, R. S., \& Abbad, G. (2005). Treinamento a distância via internet: Construção e validação de Escala de Estratégias de Aprendizagem. Anais do XXIX Encontro Nacional dos Programas de Pós-Graduação em Administração, Brasília.

Recebido em janeiro de 2008 Reformulado em junho de 2008 Aprovado em setembro de 2008

Sobre as autoras:

Thaís Zerbini é professora de Psicologia Organizacional e do Trabalho, mestre e doutora em Psicologia pela Universidade de Brasília. Integra grupos de pesquisa sobre Treinamento, Desenvolvimento e Educação de pessoas (TD\&E) e suas publicações recentes tratam de avaliação de impacto de treinamento a distância, suporte à transferência de treinamento, estratégias de aprendizagem, reações aos procedimentos instrucionais e desempenho de tutor em cursos a distância, entre outros assuntos ligados a TD\&E.

Gardênia Abbad é professora adjunta do Departamento de Psicologia Social e do Trabalho na Universidade de Brasília, mestre e doutora em Psicologia pela Universidade de Brasília. Coordena grupo de pesquisa sobre avaliação de programas de Treinamento, Desenvolvimento e Educação de Pessoas, presenciais e a distância. Suas publicações recentes tratam de avaliação de ações educacionais presenciais e a distância, entre outros assuntos ligados a TD\&E. 
